\title{
Pseudo Convex Framework using Sparse Channel Estimation for Multipath Fading Channels in DS-CDMA Systems
}

\author{
Rajendrakumar Govinda Zope, Devendra Ningappa Kyatanavar
}

\begin{abstract}
A Direct-Sequence Code-Division Multiple-Access (DS-CDMA) with rake receiver for multiuser system sets up a new dimension in mobile communication system. We propose a pseudo convex framework using an optimum sparse channel estimation technique for DS-CDMA mobile communication systems. Further, the blind channel estimation problem will be examined for rake-based DS-CDMA communication framework with time variant multi-path fading channels This receiver accomplishes an effective assessment of channel according to a maximum convexity criterion, by means of the sparse technique. This estimation method requires a convenient representation of the discrete multipath fading channel based on the sparse theory. In this paper, we have defined a specialized interior-point method for solving large-scale $\ell_{1}$ - problem in multiuser detection. Our method can be generalized to handle a variety of extensions such as various channel conditions. A new solution to DS-CDMA based sparse channel estimation is presented in this paper that assures a global optimal solution. Also, it is proven that the said solution can be used as an apparent program that shall enable solutions utilizing interior point techniques involving polynomial time complexity. Through simulation the rationality of the techniques proposed in this paper has been highlighted by results obtained for various modulation schemes and channel parameters. The execution of a pseudo convex framework using sparse channel estimation technique with rake receiver in DS-CDMA framework for multipath fading channels is explored. The overall performance is evaluated in terms of bit error rate (BER) for a range of values of signal to noise ratio (SNR). This framework gives better performance under various modulation schemes using pseudo noise (PN) spreading code. Furthermore, performance of the proposed system is compared with different detectors.
\end{abstract}

Keywords: DS-CDMA, Multipath fading channel, Rake receiver, Sparse estimation

\section{INTRODUCTION}

A typical RAKE receiver utilizes principle of multipath diversity by collecting signal energies rather than suppressing it. RAKE receiver tries acquiring the time delayed/advanced versions of the prime signal by utilizing a distinct correlation receiver referred to as finger for each multipath signal. These fingers of RAKE receiver have their unique code generators to produce diverse phases, whereby each phase resembles to different multipath.

Revised Manuscript Received on May 10, 2020.

* Correspondence Author

Rajendrakumar Govinda Zope* Department of Electronics and Telecommunication Engineering Sanjivani College of Engineering, Kopargaon-423 603 Maharashtra, India. Savitribai Phule Pune University, Pune, Maharashtra, India. Email: rgzope2002@rediffmail.com

Devendra Ningappa Kyatanavar, Department of Electronics and Telecommunication Engineering Sanjivani College of Engineering, Kopargaon-423 603 Maharashtra, India. Savitribai Phule Pune University, Pune, Maharashtra, India. Email: kyatanavar@gmail.com

(c) The Authors. Published by Blue Eyes Intelligence Engineering and Sciences Publication (BEIESP). This is an open access article under the CC BY-NC-ND license (http://creativecommons.org/licenses/by-nc-nd/4.0/)
Recent works displayed that the improvement in overall performance of traditional dielectric strength code division multiple access might be due to ratification in chaos based spread sequences. Even though aforementioned improvement is shown when the receiver doesn't have multiuser detection capabilities, the initial results obtained turn out promising.[10].

In this paper, we study and discuss distinct correlator RAKE receiver for DS-CDMA systems, whereas in typical RAKE receivers, numerous correlators combine the multipath signals then align them subsequently before taking any decision. The Global system for Mobile, also known as 2nd generation European mobile telephony standard chose Gaussian minimum shift keying (GMSK) as it was much familiar modulation scheme.

Usually, during transmission of signal from base station, all the frequency channels power amplifier outputs are multiplexed before it reaches the transmitting antenna.

The only problem with GMSK for direct sequence spread spectrum (DS-SS) is that, it being a nonlinear modulation system, the data is transferred with respect to change in carrier phase rather than the absolute phase. So, this type of schema shall witness performance degradation in firsthand. However, the data can be preprocessed in such a way that it shall be carried in absolute phase before imparting on to GMSK modulator.

Communication systems, in recent times, require appropriate concerns to utilize the fundamentally yet most basic capabilities like channel bandwidth efficiency and transmitted power [5],[6]. The GMSK proves to utilize the power and bandwidth in optimum way, hence it makes itself more suitable mobile communications [5]-[7].

Channel estimation becomes an unparallel vital strategy of wireless communication systems, as many modern communication technologies estimate readiness of channel state information. Presently, in cellular communication systems, estimation of channel is ordinarily done by utilizing pilot signal, which is generally a known signal. As this familiar signal don't convey user information but bandwidth reduction and power consumption of training signal is observed, so to retain accuracy has been one of the essential scopes on channel estimation. It seems pretty legitimate that varied non-training assisted channel estimation strategies (blind channel estimation) have been recommended, but the blind scheme is not even rarely used in real world communication system as it is needs excessive computational complexity.

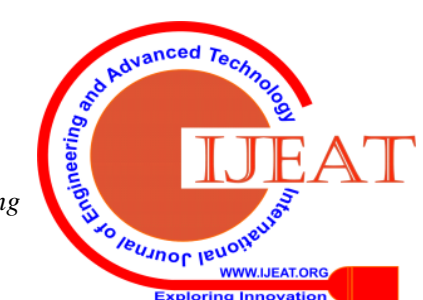


In virtue of training-based channel estimation techniques, various features of cellular channels (space, domain, frequency domain and time domain) are utilized to lessen the quantity of trained signal, one of them being sparsity of channel impulse response. It is noted that this impulse response is sparse for broad bandwidth even though the environment is scattering wealthy or not [15].

Further, in this paper, problem definition is explained in section II, while, methodology is introduced in Section III. In Section IV, results and discussion to validate the superiority of proposed system is discussed. Finally, section V concludes the proposed work of paper.

\section{PROBLEM DEFINITION}

The DS-CDMA is so attractive for cellular and personal communications, due to its potential to increase the capacity and some various other aspects like capability to fade anti-multipath signals [1]. However, systems employing DS-CDMA have demonstrated the limit of users' i.e. maximum number of users communicating simultaneously. This limitation is due to the interference among users that exists beside the channel noise [8]. The optimum detector for DS-CDMA systems, is maximum likelihood sequence estimator. However, its complexity arises exponentially with the number of users and therefore, it is unfeasible. Matched Filtering has a low complexity; however, its performance is optimum only in the case that there is no interference, and it is very poor in the case of interfering users. So, we must seek a sub-optimum detector with a lower complexity than the maximum likelihood detector and a better performance than the matched Filtering [9].

\section{A. Multi-user Detection using Blind Constant Modulus}

Consider $\mathrm{k}$ simultaneous users in an un-coded DS-CDMA system. Let $s_{k}$ represent $P x 1$ vector of the k-th users' spreading code with an assumption that codes are normalized, i.e. $\left\|s_{k}\right\|_{2}=1$

Let $b_{k}(t)$ is symbol transmitted to user $\mathrm{k}$. In multichannel communication, $\mathrm{x}(t)$ (having dimension $P \times 1$ ) being the complex envelope of the signals, received by a lone user i.e. first user, after denoising and sampling becomes,

$$
x\left(t_{n}\right)=A_{1} b_{1}\left(t_{n}\right) s_{1}+\sum_{k=2}^{K} A_{k} b_{k}\left(t_{n}\right) s_{k}+n\left(t_{n}\right) \#(1)
$$

where $t_{n}=$ Sampling time

$A_{k}=$ Received amplitude of kth user $n\left(t_{n}\right)=$ sampled noise

For multichannel communication with maximum delay of $L T_{c}$, where $T_{c}$ is chip time.

The received signal can be modelled as,

$$
\begin{gathered}
x\left(t_{n}\right)=A_{1} b_{1}\left(t_{n}\right) S_{1} h_{1}+u_{1}\left(t_{n}\right) \\
+\sum_{k=2}^{K} A_{k} b_{k}\left(t_{n}\right) S_{k} h_{k}+u_{k}\left(t_{n}\right)+n\left(t_{n}\right)^{\#(2)}
\end{gathered}
$$

where $S_{k}$ is $(P+L-1) x L$ matrix, whose columns are shifted version of spreading code vector by one chip bit delay $s_{k}$, where,

$\mathrm{h}_{\mathrm{k}}=L x 1$ vector, of $\mathrm{k}$-th user channels' response, $\mathrm{u}_{\mathrm{k}}\left(\mathrm{t}_{\mathrm{n}}\right)$ is $\mathrm{k}$-th users' Inter-symbol interference by adjacent symbols and $n\left(t_{n}\right)$ denotes noise vector.

Observing (2), problem of reconstruction of symbol of user 1 from $\mathrm{N}$ received samples is blind signal estimation and can be modelled as $y(t)=w^{H} x(t)$ which guarantees of perfect reconstruction.

Now, we have to minimize the constant modulus (CM) cost function, by calculating $w$ that minimizes the sample-average of the deviation of squared modulus of $y(t)=w^{H} x(t)$ from its supposed value, which can be randomly set to 1 is given by,

$$
\widehat{w}=\arg \min _{\mathrm{w}} \frac{1}{N} \sum_{n=1}^{N}\left(\left|w^{H} x\left(t_{n}\right)\right|^{2}-1\right)^{2} \#(4 a)
$$

Here $w$ gives the symbol of user-1, so for other user symbols linear constraint is to be established as,

$$
w^{H} C_{1}=1 \#(4 b)
$$

$$
C_{1}=S_{1} h_{1} \#(4 c)
$$

with $S_{1}$ and $h_{1}$ is mentioned in eq (2), and is likely to be identified. Notice that, when the multipath channel is equal to 1 i.e. $\mathrm{L}=1$, the limitation from (4b) and (4c) descends to,

$$
w^{H} S_{1}=1 \#(4 d)
$$

The approach formulated (4) is known as cost function of constrained constant modulus (CCM). Here, observe that vector $w$ is a minimization problem of 4-th order and does not affirm a closed form solution. Furthermore, it bears several local minima, inducing challenge to global minimization.

Subsequently, we exhibit the approximation of this CCM cost function by using convex optimization approach with sparse estimation, thus affirming global optimality [11].

\section{METHODOLOGY}

Assuming MC-DS-CDMA system, consider $J$ number of users with $j$ as reference user in an explicit cell for unspecified time period. A system model is made with certain assumptions as,

i. at any given time, all users are operating/active.

ii. the power levels of transmitter by entire users are alike.

iii. at any given time, the fading of transmitted signal is same i.e. flat.

iv. The chip rate is much smaller than the bit rate of the coded pseudo noise sequences. 
The list of notations used in the system analysis are as below:

$J=$ Total number of users

$P=$ Chip power of each user

$N_{c}=$ No. of chip code for each subcarrier channel

$N=$ No. of chips of code

$L=$ Code length of each user

Now, $j^{\text {th }}$ users' input information is modified into $N_{c}$ parallel streams of data and each of it is coded by channel's respective code. Hence, in time domain, every data bit is accelerated and modulated by corresponding sub carrier.

Generally, the sub carrier's expression can be written with following notations as,

$$
\begin{aligned}
& C^{j}=\text { Code of } j^{\text {th }} \text { user } \\
& R_{b}=\text { Bit rate } \\
& m_{j}(t)=\text { Input data Stream of } j^{\text {th }} \text { user } \\
& B_{n}^{j}(t)=n-\text { th bit } m_{j}(t) \\
& m_{j}(t)=\sum_{-\infty}^{\infty} b_{n}^{j} \text { where } b= \pm 1 \\
& \quad \omega_{c}=\text { Frequency of the reference channel } \\
& \quad \Delta \omega_{c}=\text { Freq. spacing between } 2 \text { successive channels } \\
& \emptyset_{k}=\text { Phase angle of the kth sub carrier } \\
& \text { So,the signal expression of the sub carrier is given by, }
\end{aligned}
$$

$$
\sum_{\kappa=1}^{N c} \sqrt{2 P} b_{n, k}^{j} \cos \left(\omega_{c} t+\kappa \Delta \omega_{c}+\phi_{\kappa}\right) \#(5)
$$

Thus, the j-th user transmitted signal's expression is described by,

$$
\begin{gathered}
S \tau(t)= \\
\sum_{\kappa=1}^{N c}\left\{\sqrt{2 P} b_{n, k}^{j}\left(\sum_{x=1}^{N} C_{x, k}^{j}\right) \cos \left(\omega_{c} t+\kappa \Delta \omega_{c}+\phi_{\kappa}\right)\right\} \#(6)
\end{gathered}
$$

$b_{n, k}^{j}=\mathrm{J}$ user bit for modulation by $\mathrm{k}$ channel $\mathrm{n}$-th bit of the $\mathrm{j}$-th user which is being modulated by k-th channel.

$C_{x, k}^{j}=\mathrm{x}$-th chip of $\mathrm{k}$-th section of $\mathrm{j}$-th users code [3].

A lone user of DS-CDMA rake receiver usually contains many correlators. The performance of bit error rate is determined by the number of branches occupied by the rake receiver. Seeing the constraints of hardware, generally a 4 branched rake receiver is often used.

The Nyquist filters are recommended for baseband pulse shaping as the paths can be combined unjustifiably. Such thing happens due to the alteration in the auto-correlation characteristics caused by random noise.

The system capacity is decided by the framework of rake receiver and the multiple access interference (MAI), which is a consequence of the spreading code imperfection and defective auto-correlation characteristics [4].

The lone/ solo user receiver accepts the received signals from other operating users as inactive interferences.

While in multi-user detection, the non-orthogonal characteristics of received signals are reduced by detecting the signal in an unbiased way, giving better performance. However, it is not capable to use the scattered energy up-to its full extent [2].

During reception, the receiver of $j^{\text {th }}$ user collects signals communicated by all the $j$ users. Hence, the received signal's expression is given by,

$$
r(t)=\sum_{m=1}^{j}\left[\sqrt{2 P} \sum_{k=1}^{N c} b_{n, k}^{m}\left(\sum_{x=1}^{N} C_{x, k}^{j} \cos \left(\omega_{c} t+\kappa \Delta \omega_{c} t+\phi \kappa\right)+\eta(t)\right)\right] \# \#(7)
$$

Here, $n(t)=$ channel AWGN noise, whose spectral density is around $\mathrm{N}_{0} / 2$

After considering effect of multipath Rayleigh fading,

where,

$$
r(t)=\sum_{m=1}^{j} \alpha\left[\sqrt{2 P} \sum_{k=1}^{N c} b_{n, k}^{m}\left\langle\sum_{x=1}^{N} C_{x, k}^{j}\right\rangle \cos \left(\omega_{c} t+\kappa \Delta \omega_{c} t+\theta+\phi \kappa\right)+\eta(t)\right] \#(8)
$$

$\alpha=$ Iinstaneneous amplitude distortion,

$\theta=$ Instaneneous phase distortion due fading

But, an offset in carrier frequency is observed because of Doppler Effect.

If $\delta \omega_{c}$, is the amount of such frequency offset then,

$$
r(t)=\sum_{m=1}^{j} \alpha\left[\sqrt{2 P} \sum_{k=1}^{N c} b_{n, k}^{m}\left\langle\sum_{x=1}^{N} C_{x, k}^{j}\right\rangle \cos \left(\omega_{c} t+\kappa \Delta \omega_{c} t+\theta+\phi \kappa+\delta \omega_{c} t\right)+\eta(t)\right] \#(9)
$$

After demodulation and decoding the equation will be,

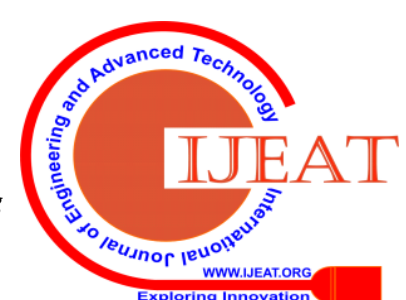




$$
\begin{gathered}
y(t)=\sum_{m=1}^{j}\left[\frac{\alpha \sqrt{2 P}}{2} \sum_{k=1}^{N_{c}} b_{n k}^{m}\left\langle\sum_{x=1}^{N}\left(C_{x, k}^{m} \times C_{x, k}^{j}\right)\right\rangle \cos \left(2 \omega_{c} t+2 k \Delta \omega_{c} t+\theta+\phi_{k}+\delta \omega_{c} t\right)+\cos \left(\delta \omega_{c} t+\phi_{k}+\theta\right)\right] \\
+\sum_{k=1}^{N_{c}} \eta(t) \sum_{x=1}^{N} C_{x, k}^{j} \cos \left(\omega_{c} t+k \Delta \omega_{c} t\right) \#(10)
\end{gathered}
$$

Separating it after cross and auto correlation

$$
\begin{gathered}
y(t)=\frac{\alpha \sqrt{2 P}}{2} \sum_{k=1}^{N_{c}}\left[b_{n k}^{j}\left\langle\sum_{x=1}^{N}(1)\right\rangle\left\{\cos \left(2 \omega_{c} t+2 k \Delta \omega_{c} t+\theta+\phi_{k}+\delta \omega_{c} t\right)+\cos \left(\delta \omega_{c} t+\phi_{k}+\theta\right)\right\}\right] \\
+\sum_{m=1}^{j=1}\left[\frac{\alpha \sqrt{2 P}}{2} \sum_{k=1}^{N_{c}} b_{n k}^{m}\left\langle\sum_{x=1}^{N}(\mu)\right\rangle\left\{\cos \left(2 \omega_{c} t+2 k \Delta \omega_{c} t+\theta+\phi_{k}+\delta \omega_{c} t\right)+\cos \left(\delta \omega_{c} t+\phi_{k}+\theta\right)\right\}\right] \\
+\sum_{k=1}^{N_{c}} \eta(t) \sum_{x=1}^{N} C_{x, k}^{j} \cos \left(\omega_{c} t+k \Delta \omega_{c} t\right) \#(11)
\end{gathered}
$$

The values of $\mu$ is " 1 " during peak interference and " 0 " when interference is absent.

Now, this shall pass through low pass filter (LPF) where the high frequency components will be rejected. Hence, the signal turns into,

$$
\begin{gathered}
y(t)=\frac{N \alpha \sqrt{2 P}}{2} \sum_{k=1}^{N_{c}} b_{n k}^{j} \cos \left(\delta \omega_{c} t+\phi_{k}+\theta\right)+ \\
\sum_{m=1}^{j-1}\left[\frac{\alpha \sqrt{2 P}}{2} \sum_{k=1}^{N_{c}}\left\{b_{n k}^{m} \mu N \cos \left(\delta \omega_{c} t+\phi_{k}+\theta\right)\right\}\right]+\sum_{k=1}^{n_{c}} \eta(t) \sum_{x=1}^{N} C_{x, k}^{j} \cos \left(\omega_{c} t+k \Delta \omega_{c} t\right) \#(12)
\end{gathered}
$$

After integrating it over 0 to $T_{b}$,

$$
\begin{aligned}
& y(t)=\frac{N \alpha \sqrt{2 P}}{2 T_{b}} \int_{0}^{T_{b}} \sum_{k=1}^{N_{c}} b_{n k}^{j} \cos \left(\delta \omega_{c} t+\phi_{k}+\theta\right) d t+ \\
& \sum_{m=1}^{j-1}\left[\frac{\alpha \sqrt{2 P}}{2 T_{b}} \int_{0}^{T_{b}} \sum_{k=1}^{N_{c}}\left\{b_{n, k}^{m} \mu N \cos \left(\delta \omega_{c} t+\phi_{k}+\theta\right)\right\}\right] d t+ \\
& \frac{1}{T_{b}} \int_{0}^{T_{b}} \sum_{k=1}^{n_{c}} \eta(t) \sum_{x=1}^{N} C_{x, k}^{j} \cos \left(\omega_{c} t+k \Delta \omega_{c} t\right) d t \#(13)
\end{aligned}
$$

Momentarily, the offset frequency is ignored, hence equation (13) becomes,

$$
\begin{aligned}
& y(t)=\frac{N \alpha \sqrt{2 P}}{2 T_{b}} \sum_{k=1}^{N_{c}} b_{n k}^{j} \cos \left(\phi_{k}+\theta\right) \int_{0}^{T_{b}} d t+ \\
& \left.\sum_{m=1}^{j-1}\left[\frac{\alpha \mu N \sqrt{2 P}}{2 T_{b}} \sum_{k=1}^{N_{c}}\left\{b_{n, k}^{m} \cos \left(\phi_{k}+\theta\right)\right\}\right] \int_{0}^{T_{b}} d t\right]+\frac{1}{T_{b}} \int_{0}^{T_{b}} \sum_{k=1}^{n_{c}} \eta(t) \sum_{x=1}^{N} C_{x, k}^{j} \cos \left(\omega_{c} t+k \Delta \omega_{c} t\right) d t
\end{aligned}
$$

Or,

$y(t)=\frac{N \alpha \sqrt{2 P}}{2} \sum_{k=1}^{N_{c}} b_{n k}^{j} \cos \left(\phi_{k}+\theta\right)+\sum_{m=1}^{j-1}\left[\frac{\alpha N \mu \sqrt{2 P}}{2} \sum_{k=1}^{N_{c}} b_{n k}^{m} \cos \left(\phi_{k}+\theta\right)\right]+\frac{1}{T_{b}} \int_{0}^{T_{b}} \sum_{k=1}^{N_{c}} C_{x, k}^{j} \cos \left(\omega_{c} t+k \Delta \omega_{c} t\right) d t \#(15)$

Or we get,

Here,

$$
y(t)=y_{j}(t)+y_{M A I}(t)+\eta(t) \#(16)
$$

$y_{j}(t)=$ Desired signal of $j^{\text {th }}$ user

$y_{\text {MAI }}(t)=$ Multiple access interference

$\eta(t)=$ Noise [3]

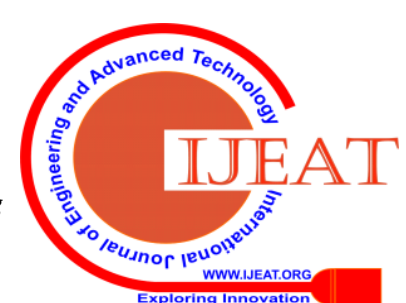




\section{A. G-MSK modulation}

Since long it is evident that G-MSK modulation has been anticipated and have been used at several places [12]. The approach, shown in Fig. 1, is analogue in nature that generates GMSK signal by applying a pre-modulation gaussian filter to the approaching binary encoded data and then to a voltage-controlled oscillator (a frequency modulator). The results obtained are said to be GMSK modulated if the input information is properly Gaussian prefiltered (bell shaped) and then applied to the Minimum Shift Keying modulator.

In this paper, we implemented it in a digital manner such that VCO is replaced by a direct digital synthesizer (DDS) along with a look-up table that substitutes the Gaussian filter [17].

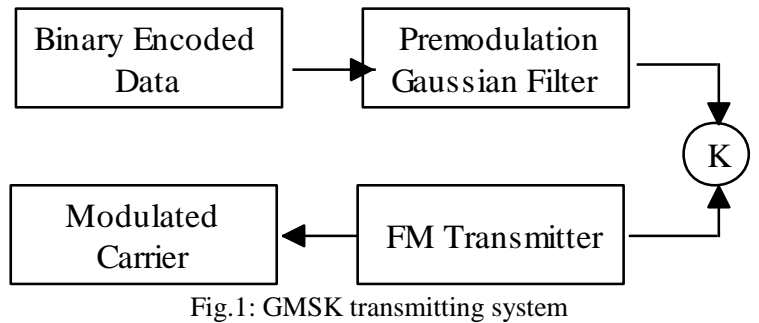

In GSM it is eminent that the Gaussian filter requires a normalized bandwidth i.e. product of bandwidth and symbol time (BxT) of 0.3 to avert inter-symbol interference (ISI). Also, it is been studied that to evaluate ISI, the interference of two adjacent bits is more than enough, which lies at either side of the currently processed bit. The gain $K$, shown in Figure 1 ensures that the modulation index $\beta$ is explicitly maintained at $1 / 2$ [16].

\section{B. Convex optimization using $\mathbf{l}_{\mathbf{1}}$-sparse estimation}

The Newton interior-point method (truncated in nature) can be prolonged to a problem in such a way that,

$$
\min \|A z-b\|_{2}^{2}+\lambda\|z\|_{1} \#(17)
$$

where, the variable $z \in C^{n}$ and the data are $b \in C^{n}$ with $A \in$ $C^{m \times n}$.

Also, $\|z\|_{1}$ is the $l_{1}$ norm of the complex vector $z$ as defined by,

$$
\|z\|_{1}=\sum_{i=1}^{n}\left(x_{i}^{2}+y_{i}^{2}\right)^{\frac{1}{2}} \#(18)
$$

where $x$ is the real part of $z$ and $y$ is the imaginary part of the received signal.

We observe that (17) can't be converted into an $l_{1}$ regularized least square of the form

$\min \|A z-b\|_{2}^{2}+\lambda\|z\|_{1}$.

Instead, it can be rewritten as the convex problem as,

$$
\text { minimise }\left\|\bar{A}\left[\frac{x}{y}\right]-\bar{b}\right\|_{2}^{2}+\lambda 1_{u}^{T} \#(
$$

subject to $\sqrt{x_{i}^{2}+y_{i}^{2}}<u_{i}, i=1, \ldots n$

where $x, y, u \in R^{n}$ are variables with the input data as,

$$
\bar{A}=\left[\begin{array}{l}
\operatorname{Re} A-\operatorname{Im} A \\
\operatorname{Im} A \operatorname{Im} A
\end{array}\right] \in R^{2 m * 2 n}, \quad \bar{b}=\left[\begin{array}{l}
\operatorname{Re} b \\
\operatorname{Im} b
\end{array}\right] \in R^{2 m}
$$

The standard barrier function for second-order cone constraints modifies the accompanying centering problem as,

$$
\begin{gathered}
\text { minimise } t\left\|\bar{A}\left[\frac{x}{y}\right]-\bar{b}\right\|_{2}^{2}+t \lambda 1^{T} \mu \\
+\sum_{i=1}^{n} \log \left(u_{i}^{2}-x_{i}^{2}-y_{i}^{2}\right) \#(20)
\end{gathered}
$$

with variables $x, y, u \in R^{n}$. The Hessian of this problem is

$$
H=t \bar{A}^{T} \bar{A}+G
$$

where $G=\nabla^{2} \sum_{i=1}^{n} \log \left(u_{i}^{2}-x_{i}^{2}-y_{i}^{2}\right)$ is the Hessian of the barrier function.

Considering the pre-conditioning such that Hessians' approximation of the first term in the objective of the centering problem (20) with its diagonal entries retains the Hessian of the logarithmic barrier as,

$$
P=t \operatorname{diag}\left(\bar{A}^{T} \bar{A}\right)+G
$$

After suitable rearrangement, this preconditioner becomes diagonal matrix block of $3 \times 3$ matrices, and hence the calculation efforts of $P^{-1} r$ is $O(n)$.This preconditioner proves to be relatively impressive when such centering problem (20) is been solved [20].

\section{RESULT AND DISCUSSION}

For Multimedia transmission using DS-CDMA application we took color videos from human action recognition videos from KTH dataset [13]. All videos are in RGB color format. Resolution of video is $180 \times 144$ and length of video for experimentation is 10 seconds. Firstly, color video is converted to binary data array using binary coder. The binary data of RGB channel is then reshape and codes sequence is applied. For experimentation we choose PN Sequence Code. The coded bits are then modulated. To test system reliability, we took GMSK Modulation scheme. Here, the uplink data is been considered, where $K$ users send DS-CDMA signals of binary form over an asynchronous multi-path having Rayleigh fading channel with AWGN. The data from multiple users thus received are subjected to individual multi-path fading channels with AWGN. At the receiver, channel is estimated using sparsed recovery with a RAKE combiner prior to the decoding stage. After decoding, we calculated BER to conclude the proposed methods accuracy.

\section{A Proposed method with GMSK modulation}

The accomplishment of DS-CDMA systems utilizing GMSK modulation has been considered. The BER values for different SNR under AWGN channel using GMSK modulation with proposed Rake receiver are as shown in Table 1.

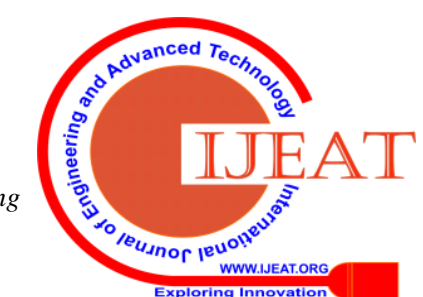




\begin{tabular}{cccccc}
\multicolumn{6}{c}{ Table 1: Average BER for different SNRs with GMSK modulation } \\
\hline $\begin{array}{c}\text { Sr. } \\
\text { No. }\end{array}$ & $\begin{array}{c}\text { SNR } \\
(\mathrm{dB})\end{array}$ & $\begin{array}{c}\text { Average } \\
\text { BER }\end{array}$ & $\begin{array}{c}\text { Sr. } \\
\text { No. }\end{array}$ & $\begin{array}{c}\text { SNR } \\
(\mathrm{dB})\end{array}$ & $\begin{array}{c}\text { Average } \\
\text { BER }\end{array}$ \\
\hline 1 & 1 & 1 & 11 & 11 & 0.502718 \\
2 & 2 & 0.957294 & 12 & 12 & 0.343384 \\
3 & 3 & 0.950906 & 13 & 13 & 0.328684 \\
4 & 4 & 0.906088 & 14 & 14 & 0.294536 \\
5 & 5 & 0.84284 & 15 & 15 & 0.246413 \\
6 & 6 & 0.774754 & 16 & 16 & 0.205792 \\
7 & 7 & 0.726843 & 17 & 17 & 0.175199 \\
8 & 8 & 0.725092 & 18 & 18 & 0.17503 \\
9 & 9 & 0.688447 & 19 & 19 & 0.112151 \\
10 & 10 & 0.654897 & 20 & 20 & 0.094569 \\
\hline
\end{tabular}

Average BER for proposed receiver for GMSK modulation scheme is as shown in Fig. 2. By expanding the SNR, BER will in general lower and reach nearer to zero for GMSK modulation method. It is noticed that, the received video quality has enhanced with increment in SNR values utilizing GMSK modulation.

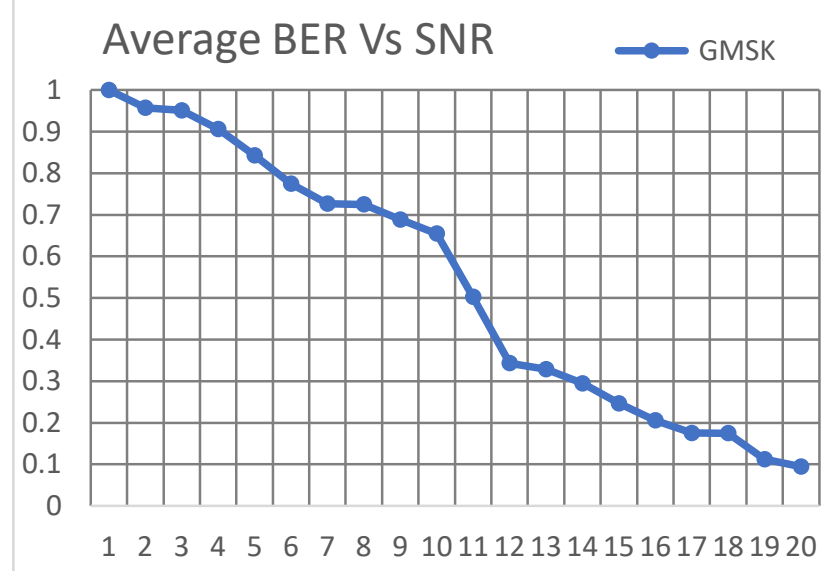

Fig. 2 Average BER Vs SNR for GMSK modulation.

\section{B. Proposed method with different modulation}

The validity of the proposed method is highlighted by simulation results obtained for the various modulation scheme. Average BER comparison for proposed rake receiver for BPSK, QPSK and GMSK modulation scheme is as shown in Fig. 3. By expanding the SNR, BER will in general diminishing and reach closer to zero for all these modulation methods.

It is noticed that, the received video quality has improved with increment in SNR values utilizing BPSK, QPSK and GMSK modulation. Likewise, it is found that average BER is 0.145321, 0.133907, 0.094569 respectively for BPSK, QPSK and GMSK modulation for proposed method when the SNR value is $20 \mathrm{db}$. Hence it very well may be inferred that DS-CDMA framework under AWGN channel with proposed receiver utilizing GMSK modulation reveals good outcomes. C. Proposed system with and without rake receiver

The validity of the proposed technique is featured by simulation results acquired with presence and absence of rake

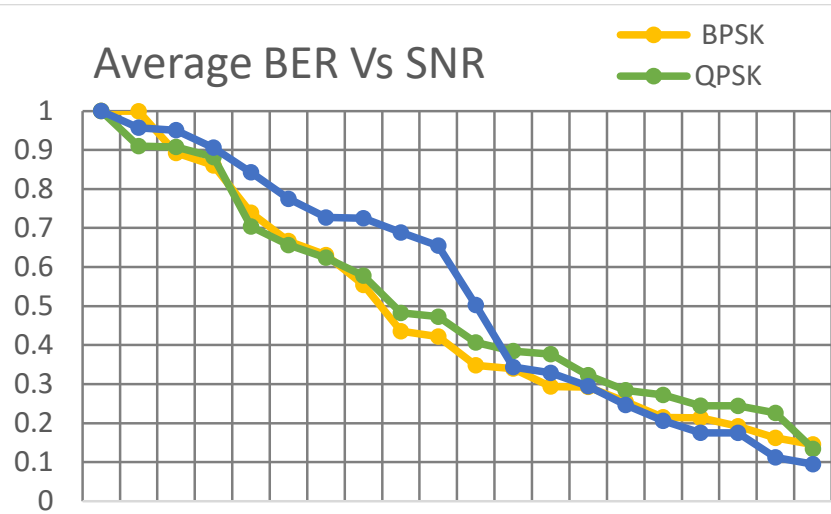

$122 \quad 3 \quad 4 \quad 5 \quad 6 \quad 7 \quad 8 \quad 9 \quad 1011121314151617181920$

Fig. 3 Average BER Vs SNR for BPSK, QPSK and GMSK modulation.

receiver. BER of rake receiver yield is estimated for different rake finger numbers (less or equal to multiple path numbers), for single-user and multi-user scenarios. Average BER comparison for proposed rake receiver and without rake receiver is as shown in Fig. 4.

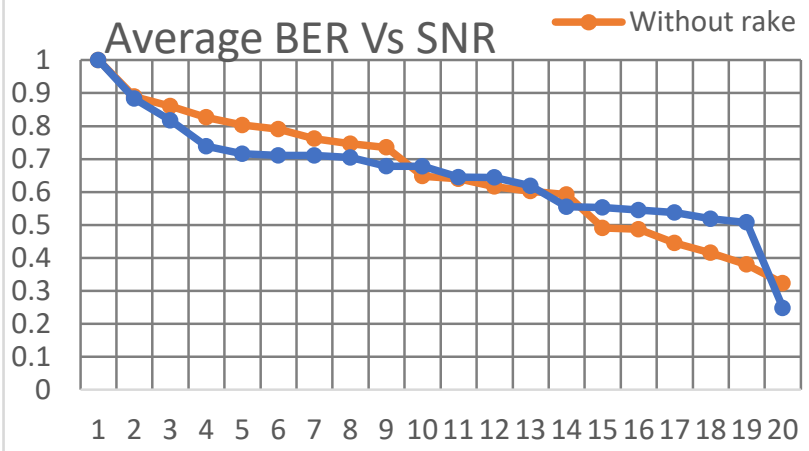

Fig.4 Comparison of BER for rake and without rake receiver.

Results of simulation show that that numbers of users result in deterioration of BER efficiency, while increasing numbers of rake fingers have better performance, It is seen that, received video quality has not improved with increment of SNR values without rake receiver and when the value of SNR is $20 \mathrm{~dB}$ then BER is 0.32339 .

With proposed rake receiver, it is seen that, the received video quality has improved essentially with the expanding SNR values. Likewise, it is found that, average BER is 0.247962 for proposed rake receiver when the SNR value is $20 \mathrm{~dB}$ Again it is concluded that. DS-CDMA system under AWGN channel with proposed rake receiver utilizing BPSK modulation reveals good outcomes.

\section{Performance comparison}

In this section, the performance of proposed system was compared by different simulation results. The comparison were done among the constrained constant modulus recursive least square detector (CCM-RLS) [19], the constrained minimum variance detector (CMV)[20], the subspace-based detector (SUB) [20], and the Kalman-filter detector (KF) [21].The performance of these systems is compared in terms of BER. Experiment evaluates the SINR (signal to noise plus interference ratio) and the bit error rate (BER) as a function of SNR (-10 dB to $25 d B)$.

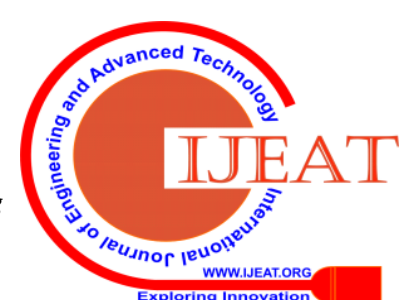


The number of users is $K=5$ and $K=15$. The simulation results are presented in Fig. 5 and Fig.6. From the simulation results it is observed that the remarkable SINR and BER performance of the proposed system are apparently true, especially for the $10 \mathrm{~dB}$ to $25 \mathrm{~dB}$ SNR.

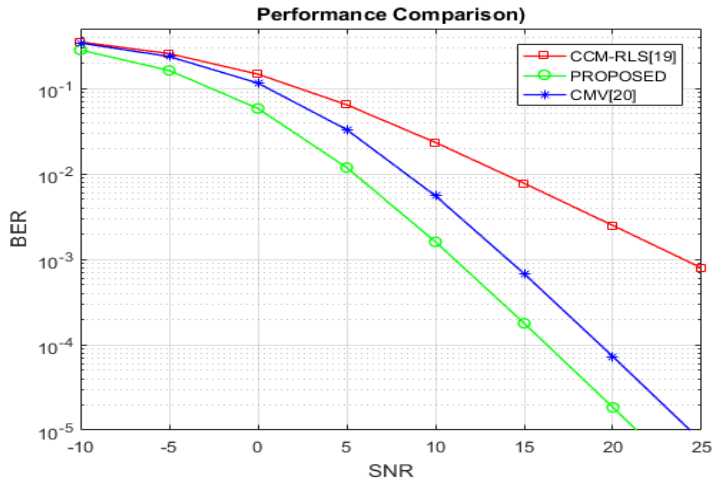

Fig. 5(a)

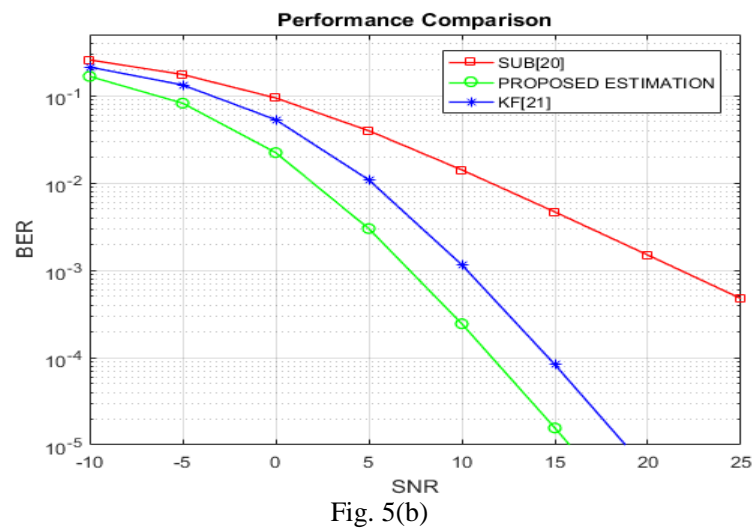

Fig. 5(a \& b) Comparison between proposed and various methods for 5 users.

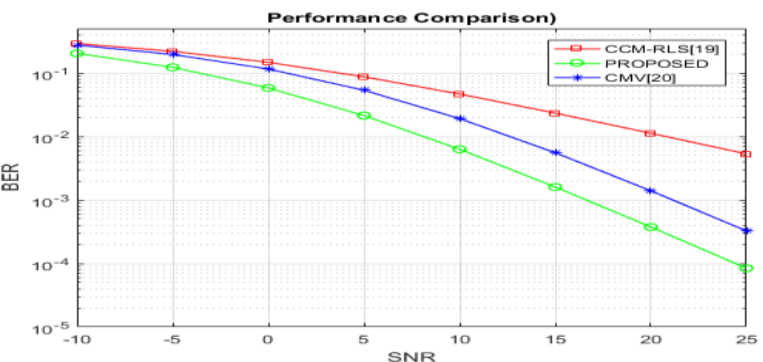

Fig. 6(a)

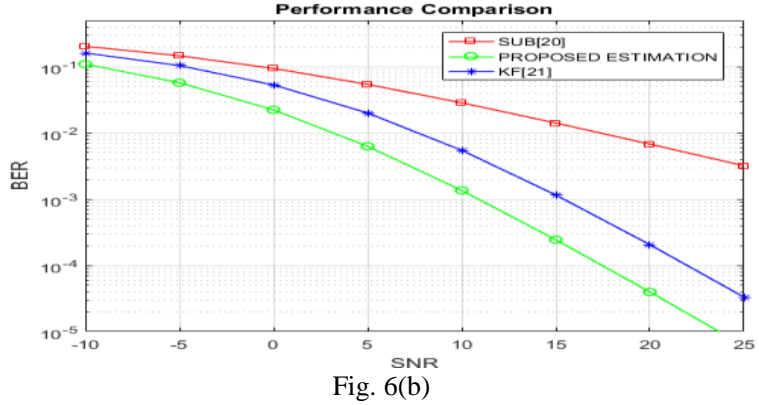

Fig.6 (a \&b) Comparison between proposed method and various methods for 15 users.

\section{E. Color video transmission}

The 1st frame of the original video is sent by means of the DS-CDMA system and the received video is obtained by changing the SNR of AWGN channel, using various modulations (BPSK, QPSK, GMSK), without rake receiver and with proposed rake receiver as shown in Fig.7.
In order to attempt to carry video by using DS-CDMA system, we choose a color trial video loaded in its three RGB components. These components value ranges already from 0 to 255 and then converted between 0 and 1 by means of binary codec. The program runs under AWGN channel using PN spreading code with various modulation schemes. The result shows that with proposed rake receiver, the quality of the received video enhanced.

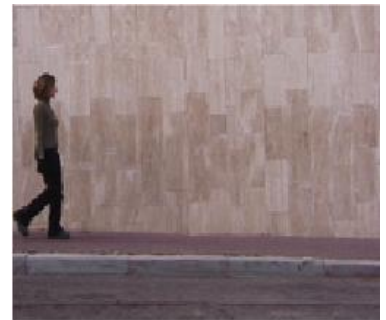

Original Video
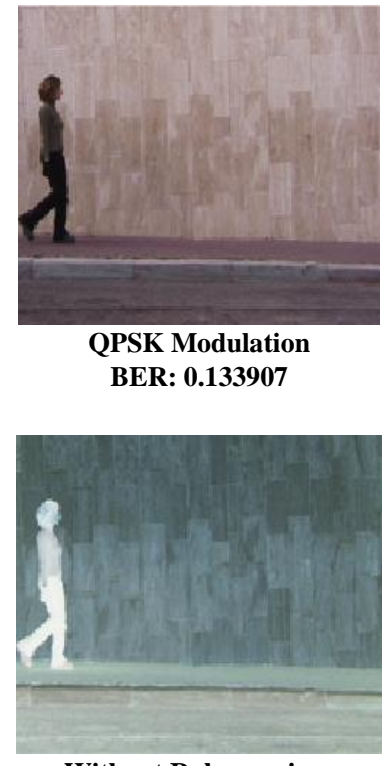

Without Rake receiver BER:0.32339
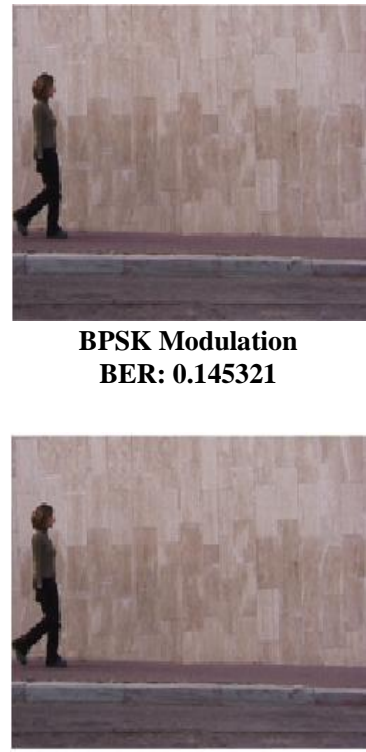

GMSK modulation BER: 0.094569

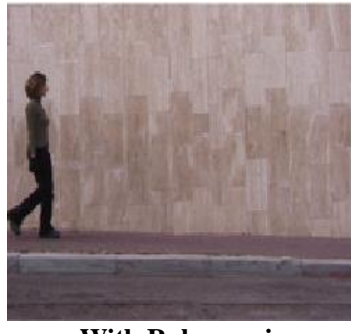

With Rake receive BER:0.247962
Fig. 7. Color video received respectively with various codes, channels, modulations and Without rake receiver and proposed rake receiver at $S N R=20 \mathrm{~dB}$.

\section{CONCLUSION}

An exceptional interior-point method for determining large-scale $\ell 1$ - channel estimation for DS -CDMA systems is been discussed. Numerical experimentations reveal that for sparse problems, the proposed method is reliable in finding accurate solutions with a computational effort that scales well with problem size, up to over one million variables. We have presented a new solution to DS-CDMA based sparse channel estimation, thus guaranteeing its global optimality. It is also been demonstrated that the proposed solution can be shaped as a semi-definite program, thus empowering the solution by interior points techniques that has polynomial time complexity. In typical DS-CDMA scenarios, experimentations were performed and the intended solutions demonstrated admirably.

Published By:

Blue Eyes Intelligence Engineering

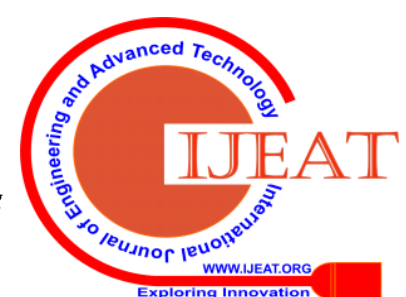


This remarkable performance is specifically apparent in challenging conditions having a smaller number of samples. The superiority in performance can be accredited to two factors: (i) the convexity of the proposed technique, which assures minimum interference, and (ii) the better performance with reducing BER, resulting in retrieving desired signal. The performance of a pseudo convex framework using sparse channel estimation technique with rake receiver in DS-CDMA framework for multipath fading channels is studied. This system gives better performance under various modulation schemes with PN spreading code. The proposed framework is additionally compared with different detectors.

\section{ACKNOWLEDGEMENT}

The authors would like to express this gratitude to Sanjivani College of Engineering, Kopargaon and Savitribai Phule Pune University, Pune for their help in this work.

\section{REFERENCES}

1. Y. Fang, G. Cai, G. Han, L. Wang and P. Chen, "Performance analysis and comparison of three multiple-access DCSK cooperative communication systems over multipath fading channels," 2017 17th International Symposium on Communications and Information Technologies (ISCIT), Cairns, QLD, 2017, pp. 1-5.

2. N. Yee, J-Linnertz and G Fettweis, $\|$ Multicarrier CDMA in indoor Wireless Radio Networks, ॥PROC of IEEE PIMRC '93, Yokohama, Japan Sept.1993 pp 0109-13

3. S. Mallick and S.P. Majumder, Performance analysis of an OFDM system in the presence of carrier frequency offset, phase noise and timing jitter over Rayleigh fading channels, \| ICECE 2008, pp. 205-210, Dec. 2008

4. http://www.wirelesscommunication.nl/reference/chaptr05/mccdma/m ccdma.htm

5. Laster, J.D.: Robust GMSK Demodulation Using Demodulator Diversity and BER Estimation. Doctor of Philosophy in Electrical Engineering, Virginia Polytechnic Institute and State University, Blacksburg, Virginia (March 1997)

6. George, G., Unnikrishnan, S.: BER Performance Evaluation of GMSK for Fading Mobile Channels. International Journal of Engineering Research and Applications (IJERA) 2(3), 375-380 (2012)

7. Murota, K., Hirade, K.: Spectrum Efficiency of GMSK Land Mobile Radio. IEEE Transactions on Vehicular Technology vt-34(2) (May 1985)

8. M. G. Rego, R. P. Ramos, R. B. Filho and C. D. Almeida, "CDMA Ad Hoc Networks in Multipath Fading Channels," 2006 IEEE Ninth International Symposium on Spread Spectrum Techniques and Applications, Manaus-Amazon, 2006, pp. 406-409.

9. M. S. Sakib and M. R. Ferdous, "Performance evaluation of MC-CDMA system for the next generation wireless networks," 2018 International Conference on Innovation in Engineering and Technology (ICIET), Dhaka, Bangladesh, 2018, pp. 1-5.

10. M. Chen and A. Burr, "Multiuser detection for uplink non-orthogonal multiple access system," in IET Communications, vol. 13, no. 19, pp. 3222-3228, 3122019.

11. A. Adler and M. Wax, "Blind Constant Modulus Multiuser Detection via Low-Rank Approximation," in IEEE Signal Processing Letters, Vol. 26, no. 9, pp. 1290-1294, Sept. 2019.

12. Murota K, Hirade K. GMSK modulation for digital mobile telephony. IEEE Transactions on Communications 1981; 29(7):1044-1050

13. Schuldt, C., Laptev, I. and Caputo, B., 2004, August. Recognizing human actions: a local SVM approach. In Proceedings of the 17th International Conference on Pattern Recognition, 2004. ICPR 2004. (Vol. 3, pp. 32-36). IEEE.

14. Linz A, Hendrickson A. Efficient implementation of an I-Q GMSK modulator. IEEE Transactions on Circuits and Systems II 1996; 43(1):14-23

15. K.Hayashi,M.Nagahara,T.Tanaka, A user's guide to compressed sensing for communications systems,IEICE Trans.Commun.Vol.P96-B ,No.3.March 2013.

16. Pasupathy S. Minimum shift keying: a spectrally efficient modulation. IEEE Communications Magazine 1979; 17(4):14-22
17. Boutin N, Porlier C, Morissette S. A digital filter-modulation combination for data transmission. IEEE Transactions on Communications 1977; COM-25(10):1242-1244.

18. E. Cand`es. Compressive sampling. In Proceedings of International Congress of Mathematics, 2006.

19. L. Wang and R. C. de Lamare, "Constrained constant modulus RLS-based blind adaptive beamforming algorithm for smart antennas,' in Proc. 4th Int. Symp. Wireless Commun. Syst., Oct. 2007, pp. 657-661

20. Seung-Jean Kim, Kwangmoo Koh, Michael Lustig, Stephen Boyd, Dimitry Gorinevsky, "An Interior-Point Method for Large-Scale

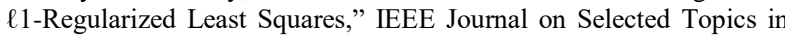
Signal Processing.

21. X. Wang and H. V. Poor, Wireless Communication Systems: Advanced Techniques for Signal Reception, 1st ed. Upper Saddle River, NJ, USA: Prentice Hall, 2004.

22. G. Yang, J. Yin, D. Huang, L. Jin, and H. Zhou, "A Kalman filter-based blind adaptive multi- user detection algorithm for underwater acoustic networks," IEEE Sens. J., vol. 16, no. 11, pp. 4023-4033, Jun. 2016

23. L. Hu, X. Zhou, and L. Zhang, "Blind multiuser detection based on Tikhonov regularization," IEEE Commun. Lett., vol. 15, no. 5, pp. 482-484, May 2011.

\section{AUTHORS PROFILE}

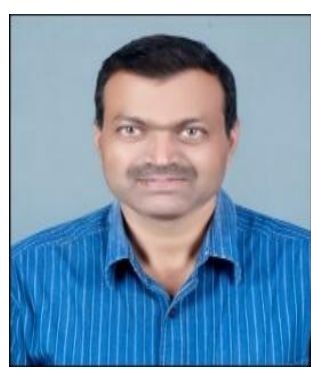

Rajendrakumar Govinda Zope was born in Buldana city Maharashtra state, India in September 1968. He has received his B.E. degree in Electronics Engineering from Amravati University, Amravati India in 1989 and $\mathrm{M}$ Tech. degree in Electronics Design and Technology from CEDT Aurangabad Dr. Babasaheb Ambedkar Marathwada University, Aurangabad, India in 1995.He is currently working as Assistant Professor in Electronics and Telecommunication Engineering Sanjivani College of Engineering Kopargaon ,Maharashtra, India His total experience in teaching 30 years. His interest is in the areas of wireless communication and Computer Network.

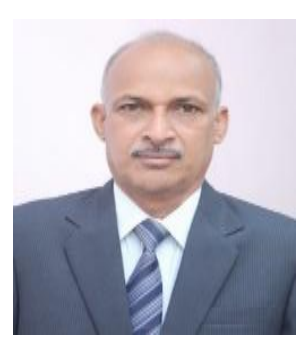

Devendra. Ningappa. Kyatanavar born at Belgaum city in Karnataka state, India. He received the Ph.D. degree in wireless communication from Shivaji University Kolhapur, Maharashtra state, India in 2009.He is currently Director, Sanjivani College of Engineering Kopargaon, Maharashtra, India. His total experience in teaching 36 years. His field of interest are Instrumentation and Control, Mobile Communication, Computer Network. 